\title{
Coexistence of stable stationary behavior and partial synchrony in an all-to-all coupled spiking neural network
}

\author{
Filip De Smet* and Dirk Aeyels ${ }^{\dagger}$ \\ SYSTeMS Research Group, Department of Electrical Energy, Systems and Automation, Ghent University, \\ Technologiepark Zwijnaarde 914, 9052 Zwijnaarde, Belgium \\ (Received 16 September 2010; revised manuscript received 12 November 2010; published 14 December 2010)
}

\begin{abstract}
We consider the stationary and the partially synchronous regimes in an all-to-all coupled neural network consisting of an infinite number of leaky integrate-and-fire neurons. Using analytical tools as well as simulation results, we show that two threshold values for the coupling strength may be distinguished. Below the lower threshold, no synchronization is possible; above the upper threshold, the stationary regime is unstable and partial synchrony prevails. In between there is a range of values for the coupling strength where both regimes may be observed. The assumption of an infinite number of neurons is crucial: simulations with a finite number of neurons indicate that above the lower threshold partial synchrony always prevails-but with a transient time that may be unbounded with increasing system size. For values of the coupling strength in a neighborhood of the lower threshold, the finite model repeatedly builds up toward synchronous behavior, followed by a sudden breakdown, after which the synchronization is slowly built up again. The "transient" time needed to build up synchronization again increases with increasing system size, and in the limit of an infinite number of neurons we retrieve stationary behavior. Similarly, within some range for the coupling strength in this neighborhood, a stable synchronous solution may exist for an infinite number of neurons.
\end{abstract}

DOI: 10.1103/PhysRevE.82.066208

PACS number(s): 05.45.Xt, 87.19.1m, 89.75.Fb, 64.60.Cn

\section{INTRODUCTION}

The power of biological neural networks, such as the human brain, has inspired scientists for decades to investigate not only how these systems may organize themselves to deal with a wide range of problems [1-6], but also how their behavior may be mimicked in applications [7-10]. While for some applications it may be sufficient to represent the communication between the neurons by continuous signals (using rate models), other phenomena-such as synchronization-rely on the discrete nature of the spikes through which the neurons interact.

The importance of the exact timing of the spikes has been demonstrated by, e.g., face recognition experiments, where the response times were too small to be resulting from a mechanism based on average spike rate, as this would require averaging over a larger time interval [11,12]. Furthermore, it has been shown that the information in the spike trains of hippocampal place cells in rats is not only encoded in the firing rate, but also in the phase with respect to rhythmical background patterns referred to as theta waves [13].

Another phenomenon where the exact spike timing is relevant is synchronization. Synchronization and time correlations between spike trains of different neurons seem to be important for grouping characteristics related to a common direction of motion, and it is generally believed that they play an important role in the phenomenon of "feature binding" [14-17], where characteristics of a common object are represented by neurons with positively correlated spike trains, allowing a representation of an object that does not require the association of a separate neuron to this object.

\footnotetext{
*fidesmet@gmail.com

†dirk.aeyels@ugent.be
}

The importance of synchronization to the functioning of the brain is also illustrated by the variety of diseases that have been shown to involve abnormal neural synchronization, such as schizophrenia, epilepsy, autism, Alzheimer disease, and Parkinson disease [18].

Analytical results on synchronization in neural systems usually deal with networks of identical neurons [19-23]. In Ref. [19] a homogeneous network of Peskin oscillators (corresponding to leaky integrate-and-fire neurons with a constant external input) [24,25] is considered, and it is shown that for almost all initial conditions, the neurons will synchronize. The authors of [20] investigated local stability properties of the synchronous solution in a heterogeneous network of a finite number of neurons. For inhibitory coupling, they showed that the synchronous solution is stable, and in case the network is strongly connected it is asymptotically stable. In Ref.[21] an overview is given of several models of an infinite number of neurons, and criteria are derived for local stability of both the stationary and (partially) synchronous solutions. In Refs.[22,23] the synchronous and asynchronous solutions in a homogeneous network of leaky integrate-and-fire neurons are investigated. The paper [22] deals with the stability of these solutions in terms of the time scale of the interactions. For slow excitatory interactions the solutions converge to an asynchronous state; for faster coupling synchrony arises, but with the individual neurons firing quasiperiodically (for systems with more than two neurons). For inhibitory interaction several synchronized clusters arise, ranging from complete synchronization for slow coupling to an asynchronous state for fast coupling. The authors of [23] analyzed the synchronous and asynchronous solutions in an all-to-all coupled network with external input spikes generated by a Poisson process. For a large range of the parameters synchronous behavior was found numerically and investigated analytically for both the supertheshold and 
the subthreshold regimes. It was also shown that the system cannot exhibit chaotic behavior.

The case of nonidentical external input currents has been investigated in several papers [26-28]. In Ref. [26] a system of leaky integrate-and-fire neurons is considered with an exponentially decaying interaction current as a response to the firing of a neuron. For weak inhomogeneity of the input currents this results in a partition of the population in a phaselocked group and a group of neurons that is moving aperiodically. An interaction type similar to the one studied in the present paper is considered in [27], which provides a sufficient criterion for full synchrony in a system of nonleaky integrate-and-fire neurons. In Ref. [28] both the cases of identical input currents and nonidentical input currents are considered for leaky integrate-and-fire neurons in the presence of noise, and it is shown that the transition from an asynchronous state to an oscillatory state corresponds to a Hopf bifurcation. For both identical and nonidentical input currents, there are parameter regions of bistability of the partially synchronous and stationary solutions when the effective interaction is inhibitory. In the present paper, we consider the noiseless case, and we investigate a similar region of bistability in terms of the coupling strength for excitatory interactions.

We study an all-to-all coupled homogeneous network (i.e., the synaptic weights are all identical) of an infinite number of nonidentical Peskin oscillators, corresponding to leaky integrate-and-fire neurons with different input currents. For a given distribution of the input currents we investigate the local stability of a stationary solution, corresponding to total incoherence, as well as the existence of a partially synchronous solution. According to the linearization of the system equations, the stationary solution is neutrally stable below a given threshold value and is unstable above this value. Synchronization may arise for values of the coupling strength above a second threshold value, smaller than the first threshold value, leading to an interval where both the stationary solution and the partially synchronous solution exist and are stable. This is confirmed by simulations of the (nonlinear) system equations, which show that, starting from an approximation of the stationary solution, the finite system always converges to the synchronized solution when it exists, but with a transient time that increases with system size.

Such transients have been studied in systems with identical neurons [29-31], for which it has been shown that the transient time increases with decreasing connectivity of the interaction network. In the present paper the connectivity is fixed to all-to-all coupling; an important aspect affecting the transient behavior is the fact that the neurons are not identical.

In Sec. II we introduce the neuron model and the system equations. Section III discusses the results concerning the stationary solution, while Sec. IV deals with the partially synchronous solution. In Sec. V we investigate the behavior of the finite system in a small region for the coupling strength around the smallest threshold value, and we explain how the system may repeatedly switch between slowly building up synchronization and a breakdown of this synchronous behavior.

\section{PRELIMINARIES}

The dynamics of a Peskin oscillator [24] below the threshold is described by

$$
\frac{d U}{d t}=I_{\mathrm{tot}}-\frac{U}{\tau},
$$

where $\tau$ is a time constant, $I_{\text {tot }}$ is the total input current, and $U$ represents the membrane potential, shifted in order to have a resting potential (i.e., the long-term potential in the absence of any input current) equal to zero, and rescaled with a factor such that the threshold corresponds to $U=1$. When $U$ reaches 1 (or becomes larger than 1 as a result of a Dirac pulse in $\left.I_{\text {tot }}\right)$, it is reset to zero, and the neuron fires a spike. Any remaining charge at the time of firing (resulting from other Dirac pulses in $I_{\text {tot }}$ at this time instance) is discarded. This may be regarded as a result of refractoriness, i.e., an absent or reduced response to input in a time interval after the spike. Although the interval length is reduced to zero in this model, the refractoriness is still relevant; all neurons spiking at the same time instance $t_{i}$ will satisfy $\lim _{t \rightarrow t_{i}}^{>} U(t)=0$, no matter whether any "order" among the spikes (due to causality) may be established.

When considering interactions between neurons in a network, the input current $I_{\text {tot }}$ is the sum of an external input $I$ (which we will assume to be constant) and an interaction current $I_{\text {int }}$. Since we will consider an all-to-all network structure, the interaction current $I_{\text {int }}$ can be taken equal for all neurons (taking into account the refractoriness, implying that a neuron will not respond to its own spikes). We will assume that the external input current $I$ is drawn from a distribution with a continuous density function $g$, with $g(I)$ equal to zero for $I \leq 1 / \tau$ (implying that for $I_{\text {int }}=0$ each neuron for which $g(I)>0$ will spike eventually) and with a finite average current $\langle I\rangle \triangleq \int_{\operatorname{supp}(g)} \operatorname{Ig}(I) d I$. (The latter condition is needed to allow for a finite interaction current $I_{\text {int }}$ since, for large $I$ values, the spiking frequency is proportional to $I$-see also Remark 4.)

The interaction current $I_{\text {int }}$ consists of a sum of Dirac pulses at the spiking times $t_{i}$, each weighted with the coupling strength $\epsilon>0$, and normalized to $N$, where $N$ denotes the number of neurons in the network:

$$
I_{\text {int }}(t)=\frac{\epsilon}{N} \sum_{i} \delta\left(t-t_{i}\right) .
$$

(A spike time may occur more than once if multiple neurons spike at the same time.)

Remark 1. Notice that for this all-to-all coupled excitatory network the normalization by $N$ is quite "natural" to obtain dynamical behavior that is (at least in some aspects) independent of the system size. However, the assumption of allto-all coupling is of course rather artificial (but more convenient for the analysis), and it has been argued that sparsely coupled networks with excitation balanced by inhibitory interactions are more appropriate to explain the irregular spiking behavior observed in experiments [32,33]. For these systems the coupling strength scales with $1 / \sqrt{K}$, where $K$ denotes the average number of connections to a neuron. 
Considering the limit $N \rightarrow \infty$, the interaction current may be written as

$$
I_{\text {int }}(t)=\epsilon \int_{\operatorname{supp}(g)} d I g(I) \rho(1-, I, t) v(1, I, t) .
$$

The expression $\rho(U, I, t)$ represents the density with respect to $U$ of the neurons with external input current $I$ at time instance $t$; it satisfies $\int_{-\infty}^{+\infty} d U \rho(U, I, t)=1$ for all possible $I$ and $t$. We use the notation $1-$ to denote the limit for $U$ to 1 from below, as the density will not be continuous at $U=1$ since it is zero for $U$ larger than 1. [Notice that for an appropriate initial condition, $\rho(U, I, t)$ will also be zero for $U<0$. It may have been different from zero for $U<0$ if the coupling strength $\epsilon$ were negative or if $g(I)$ were nonzero for negative $I$ values.] The expression $v(U, I, t)$ represents the time derivative of the membrane potential (i.e., the value of $\frac{d U}{d t}$ in the finite model) of a neuron with membrane potential $U$ and external input $I$ at time $t$ :

$$
v(U, I, t)=I+I_{\mathrm{int}}(t)-\frac{U}{\tau} .
$$

The product $\rho(1-, I, t) v(1, I, t)$ corresponds to the rate at which neurons with external input $I$ cross the threshold at time instance $t$. If there is no synchronization, this rate may be a continuous function of time. For the synchronous solution, Dirac pulses will appear in this expression and, as we will argue later on, the density $\rho(U, I, t)$ may no longer be (piecewise) continuous in $U$, but again a sum of Dirac pulses (with respect to $U$ ).

For the stationary solution we assume that $I_{\text {int }}$ does not contain Dirac pulses, and we propose the following partial differential equation for the evolution of $\rho$ :

$$
\begin{aligned}
\frac{\partial \rho}{\partial t}(U, I, t)= & -\frac{\partial(\rho v)}{\partial U}(U, I, t)+[\delta(U)-\delta(U-1)] \\
& \times \rho(1-, I, t) v(1, I, t),
\end{aligned}
$$

which is basically a continuity equation for $\rho$, expressing that the neurons crossing the threshold at $U=1$ reappear at $U=0$. Notice that, since $\rho$ is assumed to satisfy $\rho(U, I, t)=0$ for $U>1$, the Dirac pulse at $U=1$ is compensated by the derivative of $\rho v$ at $U=1$, and therefore the right-hand side of Eq. (2) may only contain a Dirac pulse at $U=0$.

\section{STATIONARY SOLUTION}

For convenience, we will assume that the density $\rho(U, I, t)$ is continuously differentiable with respect to $U$ for $U$ in the open interval $(0,1)$. This implies that the right-hand side of Eq. (2) will not contain Dirac pulses, since a Dirac pulse originating for $U=0$ would lead to a discontinuity in $\rho$ that would propagate into the interval $(0,1)$ by dynamics $(1)$.

Since $\epsilon$ - and therefore also $I_{\text {int }}$-is positive, and $g(I)$ is zero for $I$ smaller than $1 / \tau, v(0, I, t)$ is positive for all $I$ in $\operatorname{supp}(g)$ and $t \in \mathbb{R}$, and the membrane potential of a neuron cannot become negative. We may therefore assume that $\rho(U, I, t)=0$ for all $U$ outside the interval $[0,1]$, e.g., by imposing that $\rho(U, I, 0)=0$ for $U \notin[0,1]$. Consequently we may restrict our investigation to the interval $[0,1]$ for $U$, and we may replace Eq. (2) with

$$
\frac{\partial \rho}{\partial t}(U, I, t)=-\frac{\partial(\rho v)}{\partial U}(U, I, t),
$$

restricted to $U \in[0,1]$, and with the boundary condition $\rho(0, I, t) v(0, I, t)=\rho(1, I, t) v(1, I, t)$ to ensure the continuity of $\rho(U, I, t)$ in $U$. (Since $\rho(U, I, t)$ is no longer defined for $U \notin[0,1]$, the notation $\rho(1, I, t)$ is unambiguous.)

Remark 2. In Ref. [28] the authors studied an equation similar to Eq. (3), with an extra second-order term related to the noise included in their model. Because of the presence of this term, their analysis involves confluent hypergeometric functions, while the results of the present paper are expressed in terms of elementary functions. Although considering the limit of zero noise in [28] leads to similar expressions, we prefer to derive our results from Eq. (3) for convenience of the readers unfamiliar with [28] or with confluent hypergeometric functions.

\section{A. Solution structure}

We consider the stationary solution $\rho_{0}(U, I)$ of Eq. (3), obtained by putting $\frac{\partial \rho}{\partial t}$ equal to zero:

$$
\frac{\partial\left(\rho_{0} v_{0}\right)}{\partial U}(U, I)=0
$$

for all $U$ in $[0,1]$ and $I$ in $\operatorname{supp}(g)$. The expression $v_{0}(U, I)$ $=I+I_{\mathrm{int}, 0}-\frac{U}{\tau}$ represents the corresponding stationary version of $v(U, I, t)$, with $I_{\text {int }, 0}$ denoting the constant interaction current. Equation (4) implies that

$$
\rho_{0}(U, I)=\frac{C(I)}{I+I_{\mathrm{int}, 0}-\frac{U}{\tau}},
$$

for all $I$ in $\operatorname{supp}(g)$, for some expression $C(I)$ independent of $U$. Notice that $\rho_{0}$ also satisfies the boundary condition. The expression $C(I)$ is determined by the normalization of $\rho_{0}$ :

$$
1=\int_{0}^{1} \rho_{0}(U, I) d U=-C(I) \tau \ln \left(1-\frac{1}{\tau\left(I+I_{\mathrm{int}, 0}\right)}\right),
$$

or

$$
C(I)=\frac{-1}{\tau \ln \left(1-\frac{1}{\tau\left(I+I_{\mathrm{int}, 0}\right)}\right)} .
$$

The interaction current $I_{\text {int }, 0}$ is defined by the implicit equation

$$
I_{\mathrm{int}, 0}=\epsilon \int_{\operatorname{supp}(g)} d I g(I) \rho_{0}(1, I)\left(I+I_{\mathrm{int}, 0}-\frac{1}{\tau}\right),
$$

or

$$
I_{\mathrm{int}, 0}=\epsilon \int_{\operatorname{supp}(g)} \frac{-g(I) d I}{\tau \ln \left(1-\frac{1}{\tau\left(I+I_{\mathrm{int}, 0}\right)}\right)} .
$$




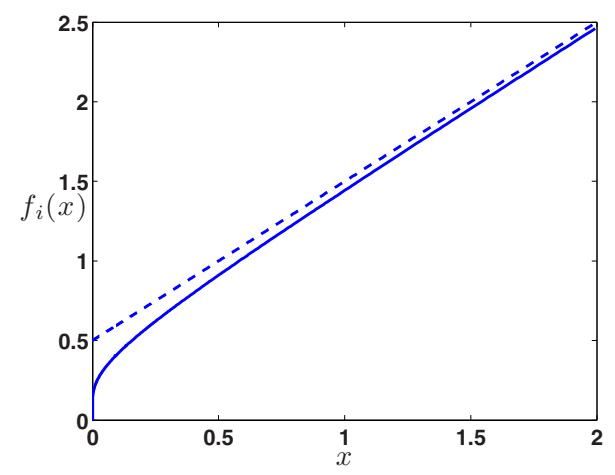

FIG. 1. (Color online) Comparison of $f_{1}(x) \triangleq \frac{-1}{\ln \left(1-\frac{1}{1+x}\right)}$ (solid line) with $f_{2}(x) \triangleq x+\frac{1}{2}$ (dashed line) for $x>0$.

Remark 3. Because of our assumptions on $\epsilon$ and $g$ it follows that $\epsilon \int_{\operatorname{supp}(g)} d I g(I) \rho_{0}(1, I)\left(I-\frac{1}{\tau}\right)>0$, and using Eq. (6) we derive that

$$
\epsilon \int_{\operatorname{supp}(g)} d \operatorname{Ig}(I) \rho_{0}(1, I)<1 .
$$

Since $\rho_{0}(1, I)>1$ as $\rho_{0}(U, I)$ is increasing in $U$ and normalized to 1] it follows that $\epsilon<1$. We conclude that for $\epsilon \geq 1$ the stationary solution does not exist.

Remark 4. The solution to Eq. (7) may be approximated using

$$
\frac{-1}{\ln \left(1-\frac{1}{1+x}\right)} \approx x+\frac{1}{2},
$$

for $x$ sufficiently large, as is illustrated in Fig. 1. Using this approximation, we obtain

$$
I_{\mathrm{int}, 0} \approx \epsilon \int_{\operatorname{supp}(g)} d I g(I)\left(I+I_{\mathrm{int}, 0}-\frac{1}{2 \tau}\right)=\epsilon\left(\langle I\rangle+I_{\mathrm{int}, 0}-\frac{1}{2 \tau}\right),
$$

and therefore

$$
I_{\mathrm{int}, 0} \approx \frac{\epsilon}{1-\epsilon}\left(\langle I\rangle-\frac{1}{2 \tau}\right)
$$

which is of course only valid for $\epsilon<1$. (See also the previous remark.)

\section{B. Spectrum}

In order to investigate the stability of the stationary solution we consider a linearization of Eqs. (3) and (6) about $\rho_{0}$ and $I_{\text {int }, 0}$ by setting

$$
\begin{gathered}
\rho(U, I, t)=\rho_{0}(U, I)+\rho_{1}(U, I, t), \\
I_{\text {int }}(t)=I_{\text {int }, 0}+I_{\text {int }, 1}(t),
\end{gathered}
$$

with $\int_{0}^{1} d U \rho_{1}(U, I, t)=0$ for all $I$ in $\operatorname{supp}(g)$ and $t$ in $\mathrm{R}$, and neglecting second-order terms, resulting in

$$
\begin{aligned}
\frac{\partial \rho_{1}}{\partial t}(U, I, t)= & -\frac{\partial}{\partial U}\left[\rho_{0}(U, I) I_{\mathrm{int}, 1}(t)\right. \\
& \left.+\rho_{1}(U, I, t)\left(I+I_{\mathrm{int}, 0}-\frac{U}{\tau}\right)\right],
\end{aligned}
$$

for all $U, t$ in $\mathrm{R}$, and $I$ in $\operatorname{supp}(g)$, and

$$
\begin{aligned}
I_{\mathrm{int}, 1}(t)= & \epsilon \int_{\operatorname{supp}(g)} d I g(I)\left[\rho_{0}(1, I) I_{\mathrm{int}, 1}(t)\right. \\
& \left.+\rho_{1}(1, I, t)\left(I+I_{\mathrm{int}, 0}-\frac{1}{\tau}\right)\right] .
\end{aligned}
$$

The boundary condition becomes (in first order)

$$
\begin{aligned}
& \rho_{0}(0, I) I_{\mathrm{int}, 1}(t)+\rho_{1}(0, I, t)\left(I+I_{\mathrm{int}, 0}\right) \\
& \quad=\rho_{0}(1, I) I_{\mathrm{int}, 1}(t)+\rho_{1}(1, I, t)\left(I+I_{\mathrm{int}, 0}-\frac{1}{\tau}\right),
\end{aligned}
$$

for all $I$ in $\operatorname{supp}(g)$ and $t$ in $\mathrm{R}$.

Because of Eq. (8), $I_{\text {int, } 1}(t)$ can be calculated explicitly from Eq. (11) as a (linear) function of $\rho_{1}(1, I, t)$. With this in mind, the right-hand side of Eq. (10) may be considered as resulting from the action of a stationary linear operator $\mathcal{L}$ on $\rho_{1}(U, I, t)$ :

$$
\frac{\partial \rho_{1}}{\partial t}=\mathcal{L} \rho_{1}
$$

To investigate the stability properties of the linearization (10) we will look at the spectrum of the operator $\mathcal{L}$ in the class of (time-invariant) functions $\widetilde{\rho}(U, I)$ (defined for $(U, I)$ in $[0,1] \times \operatorname{supp}(g))$ satisfying $\int_{0}^{1} d U \widetilde{\rho}(U, I)=0$ and the boundary condition (12) [34]. Notice that the boundary condition may also be written as

$$
\int_{0}^{1} d U \mathcal{L} \widetilde{\rho}(U, I)=0 .
$$

\section{Continuous spectrum}

The continuous spectrum of $\mathcal{L}$ contains the values of $\lambda$ $\in \mathbb{C}$ which are not eigenvalues of $\mathcal{L}$ and for which the range of $\mathcal{L}-\lambda$ is not closed, i.e., there exist functions $\tilde{\rho}_{n}$ and $f_{n}$ with $f_{n} \rightarrow f$, for some function $f$, such that

$$
\mathcal{L} \tilde{\rho}_{n}-\lambda \tilde{\rho}_{n}=f_{n},
$$

but

$$
\mathcal{L} \tilde{\rho}-\lambda \tilde{\rho}=f
$$

is not solvable to $\tilde{\rho}$, because $\tilde{\rho}_{n}$ does not converge to an appropriate function for $n \rightarrow \infty$. Since we consider functions $\tilde{\rho}_{n}$ satisfying $\int_{0}^{1} d U \widetilde{\rho}_{n}(U, I)=0$ as well as $\int_{0}^{1} d U \mathcal{L} \widetilde{\rho}_{n}(U, I)=0$, it follows that the functions $f_{n}$ (and therefore also $f$ ) satisfy $\int_{0}^{1} d U f_{n}(U, I)=0$. We will therefore investigate when Eq. (15), with $\int_{0}^{1} d U f(U, I)=0$, cannot be solved for $\tilde{\rho}$, with $\int_{0}^{1} d U \widetilde{\rho}(U, I)=0$ and $\int_{0}^{1} d U \mathcal{L} \tilde{\rho}(U, I)=0$. [Notice that only one of these conditions has to be imposed, because of $\int_{0}^{1} d U f(U, I)=0$ and Eq. (15).] 
Inspired by Eq. (11) we set

$$
\tilde{I}_{\text {int }, 1}(\widetilde{\rho}) \triangleq \frac{\epsilon \int_{\operatorname{supp}(g)} d I g(I) \widetilde{\rho}(1, I)\left(I+I_{\mathrm{int}, 0}-\frac{1}{\tau}\right)}{1-\epsilon \int_{\operatorname{supp}(g)} d I g(I) \rho_{0}(1, I)},
$$

and Eq. (15) becomes

$$
\begin{gathered}
-\frac{\partial \rho_{0}}{\partial U}(U, I) \tilde{I}_{\mathrm{int}, 1}(\widetilde{\rho})-\frac{\partial \widetilde{\rho}}{\partial U}(U, I)\left(I+I_{\mathrm{int}, 0}-\frac{U}{\tau}\right) \\
+\left(\frac{1}{\tau}-\lambda\right) \widetilde{\rho}(U, I)=f(U, I),
\end{gathered}
$$

which we rewrite as

$$
\begin{aligned}
& \frac{\partial}{\partial U}\left[\tilde{\rho}(U, I)\left(I+I_{\mathrm{int}, 0}-\frac{U}{\tau}\right)^{1-\lambda \tau}\right] \\
& \quad=\left(-\frac{\partial \rho_{0}}{\partial U}(U, I) \widetilde{I}_{\mathrm{int}, 1}(\widetilde{\rho})-f(U, I)\right)\left(I+I_{\mathrm{int}, 0}-\frac{U}{\tau}\right)^{-\lambda \tau} .
\end{aligned}
$$

Integrating over the interval $(U, 1)$, assuming that $\lambda \tau \neq-1$ (notice that this case is not important for our stability analysis) and using expression (5) for $\rho_{0}$, we obtain

$$
\begin{aligned}
\widetilde{\rho}(U, I)= & -\frac{C(I) \tilde{I}_{\mathrm{int}, 1}(\widetilde{\rho})}{\lambda \tau+1}\left(I+I_{\mathrm{int}, 0}-\frac{U}{\tau}\right)^{-2} \\
& +\frac{C(I) \tilde{I}_{\mathrm{int}, 1}(\widetilde{\rho})}{\lambda \tau+1} \frac{\left(I+I_{\mathrm{int}, 0}-\frac{U}{\tau}\right)^{\lambda \tau-1}}{\left(I+I_{\mathrm{int}, 0}-\frac{1}{\tau}\right)^{\lambda \tau+1}} \\
& +\mathcal{F}(U, I)+\widetilde{\rho}(1, I)\left(\frac{I+I_{\mathrm{int}, 0}-\frac{U}{\tau}}{I+I_{\mathrm{int}, 0}-\frac{1}{\tau}}\right)^{\lambda \tau-1},
\end{aligned}
$$

where

$$
\begin{aligned}
\mathcal{F}(U, I) \triangleq & \left(I+I_{\mathrm{int}, 0}-\frac{U}{\tau}\right)^{\lambda \tau-1} \int_{U}^{1} d U^{\prime} f\left(U^{\prime}, I\right) \\
& \times\left(I+I_{\mathrm{int}, 0}-\frac{U^{\prime}}{\tau}\right)^{-\lambda \tau} .
\end{aligned}
$$

Expressing $\int_{0}^{1} d U \widetilde{\rho}(U, I)=0$, it follows that (assuming that $\lambda \tau \neq 0$-we will see that this does not affect the stability analysis as we will show that the continuous spectrum is purely imaginary)

$$
\begin{aligned}
0= & -\frac{C(I) \tilde{I}_{\mathrm{int}, 1}(\widetilde{\rho})}{\lambda \tau+1} \tau\left[\left(I+I_{\mathrm{int}, 0}-\frac{1}{\tau}\right)^{-1}-\left(I+I_{\mathrm{int}, 0}\right)^{-1}\right] \\
& +\frac{C(I) \tilde{I}_{\mathrm{int}, 1}(\widetilde{\rho})}{\lambda \tau+1} \frac{\left(I+I_{\mathrm{int}, 0}\right)^{\lambda \tau}-\left(I+I_{\mathrm{int}, 0}-\frac{1}{\tau}\right)^{\lambda \tau}}{\lambda\left(I+I_{\mathrm{int}, 0}-\frac{1}{\tau}\right)^{\lambda \tau+1}} \\
& +\int_{0}^{1} d U \mathcal{F}(U, I)+\widetilde{\rho}(1, I) \frac{\left(I+I_{\mathrm{int}, 0}\right)^{\lambda \tau}-\left(I+I_{\mathrm{int}, 0}-\frac{1}{\tau}\right)^{\lambda \tau}}{\lambda\left(I+I_{\mathrm{int}, 0}-\frac{1}{\tau}\right)^{\lambda \tau-1}}
\end{aligned}
$$

for all $I$ in $\operatorname{supp}(g)$. If

$$
\left(I_{\mathrm{C}}+I_{\mathrm{int}, 0}\right)^{\lambda \tau}-\left(I_{\mathrm{C}}+I_{\mathrm{int}, 0}-\frac{1}{\tau}\right)^{\lambda \tau}=0,
$$

for some $I_{\mathrm{C}}$ in $\operatorname{supp}(g)$, then $\widetilde{I}_{\text {int, } 1}(\widetilde{\rho})$ can be calculated in terms of $\mathcal{F}\left(U, I_{\mathrm{C}}\right)$ [or $\left.f\left(U, I_{\mathrm{C}}\right)\right](U \in[0,1])$ using the previous equation. If this holds for more than one value of $I_{\mathrm{C}}$, then in general the obtained values for $\widetilde{I}_{\text {int }, 1}(\widetilde{\rho})$ will be different, and therefore there is no solution for $\tilde{\rho}$ to Eq. (15). Since $f$ may be approximated by functions $f_{n}$, which are defined appropriately in neighborhoods of the values $I_{\mathrm{C}}$ in order to avoid the previous contradiction, it follows that the corresponding value for $\lambda$ is in the continuous spectrum of $\mathcal{L}$.

Now assume that Eq. (17) holds for exactly one value of $I_{\mathrm{C}}$. For all $I \neq I_{\mathrm{C}}, \widetilde{\rho}(1, I)$ can be written in terms of $\widetilde{I}_{\text {int, } 1}(\widetilde{\rho})$ and $\mathcal{F}$, and substitution in Eq. (16) then leads to

$$
\begin{aligned}
\widetilde{I}_{\mathrm{int}, 1}(\widetilde{\rho})= & \frac{\epsilon}{1-\epsilon \int_{\operatorname{supp}(g)} d I g(I) \rho_{0}(1, I)} \int_{\operatorname{supp}(g)} d I g(I) \\
& \times\left(-\frac{\rho_{0}(1, I) \widetilde{I}_{\mathrm{int}, 1}(\widetilde{\rho})}{\lambda \tau+1}\right. \\
& +\frac{\lambda \tau \tilde{\mathrm{I}}_{\mathrm{int}, 1}(\widetilde{\rho})\left[\rho_{0}(1, I)-\rho_{0}(0, I)\right]\left(I+I_{\mathrm{int}, 0}-\frac{1}{\tau}\right)^{\lambda \tau}}{(\lambda \tau+1)\left[\left(I+I_{\mathrm{int}, 0}\right)^{\lambda \tau}-\left(I+I_{\mathrm{int}, 0}-\frac{1}{\tau}\right)^{\lambda \tau}\right]} \\
& -\frac{\lambda \int_{0}^{1} d U \mathcal{F}(U, I)\left(I+I_{\mathrm{int}, 0}-\frac{1}{\tau}\right)^{\lambda \tau}}{\left(I+I_{\mathrm{int}, 0}\right)^{\lambda \tau}-\left(I+I_{\mathrm{int}, 0}-\frac{1}{\tau}\right)^{\lambda \tau}} .
\end{aligned}
$$

This equation also depends on values of $\mathcal{F}(U, I)$ or $f(U, I)$ for $I \neq I_{\mathrm{C}}$, and the previously obtained value for $\widetilde{I}_{\text {int, } 1}(\widetilde{\rho})$ will generally not satisfy this equation. Similarly as before, it follows that $\lambda$ is in the continuous spectrum of $\mathcal{L}$. 
On the other hand, if there are no values $I_{\mathrm{C}}$ in $\operatorname{supp}(g)$ satisfying Eq. (17), then the linear Eq. (18) can be solved for $\widetilde{I}_{\text {int, }, 1}(\widetilde{\rho})$ unless its coefficient is zero, in which case

$$
\begin{aligned}
1- & \epsilon \int_{\operatorname{supp}(g)} d I g(I) \rho_{0}(1, I)+\frac{\epsilon}{\lambda \tau+1} \int_{\operatorname{supp}(g)} d I g(I) \\
& \times\left(\frac{\left[\rho_{0}(1, I)-\rho_{0}(0, I)\right]\left(I+I_{\mathrm{int}, 0}-\frac{1}{\tau}\right)^{\lambda \tau}}{\left.\rho_{0}(1, I)-\lambda \tau \frac{1}{\left(I+I_{\mathrm{int}, 0}\right)^{\lambda \tau}-\left(I+I_{\mathrm{int}, 0}-\frac{1}{\tau}\right)^{\lambda \tau}}\right)}\right. \\
& =0,
\end{aligned}
$$

or

$$
\begin{aligned}
& \frac{\epsilon \lambda \tau}{\lambda \tau+1} \int_{\text {supp }(g)} d I g(I) C(I) \\
& \quad \times\left(\frac{\left(I+I_{\mathrm{int}, 0}\right)^{-\lambda \tau-1}-\left(I+I_{\mathrm{int}, 0}-\frac{1}{\tau}\right)^{-\lambda \tau-1}}{\left(I+I_{\mathrm{int}, 0}\right)^{-\lambda \tau}-\left(I+I_{\mathrm{int}, 0}-\frac{1}{\tau}\right)^{-\lambda \tau}}\right) \\
& =1 .
\end{aligned}
$$

As we will see in the next section, Eq. (19) determines the eigenvalues and therefore the point spectrum of $\mathcal{L}$. We conclude that the continuous spectrum consists of (apart from the values $-1 / \tau$ and zero) the values of $\lambda \in \mathrm{C}_{0}$ for which ( $I$ $\left.+I_{\text {int }, 0}\right)^{\lambda \tau}-\left(I+I_{\text {int }, 0}-\frac{1}{\tau}\right)^{\lambda \tau}=0$ for some $I$ in $\operatorname{supp}(g)$, or

$$
\lambda \tau=\frac{2 \pi i k}{\ln \left(\frac{I+I_{\mathrm{int}, 0}}{I+I_{\mathrm{int}, 0}-\frac{1}{\tau}}\right)},
$$

for some $k$ in $Z_{0}$ and some $I$ in $\operatorname{supp}(g)$. Since the continuous spectrum is nonempty and lies on the imaginary axis, it follows that-according to the linearization - the stationary solution cannot be asymptotically stable, and if there are no eigenvalues with a positive real part it is neutrally stable.

\section{Point spectrum}

The point spectrum consists of the eigenvalues of $\mathcal{L}$, which are the values of $\lambda \in \mathrm{C}$ for which

$$
\mathcal{L} \tilde{\rho}-\lambda \widetilde{\rho}=0
$$

has a nonzero solution for $\tilde{\rho}$. Since this equation corresponds to Eq. (15) with $f=0$, we can repeat the calculations from the previous section with $\mathcal{F}=0$; and, imposing that $\left(I+I_{\text {int }, 0}\right)^{\lambda \tau}$ $-\left(I+I_{\text {int, } 0}-\frac{1}{\tau}\right)^{\lambda \tau}$ is nonzero for all $I$ in $\operatorname{supp}(g)$, we now require that Eq. (19) is satisfied to allow for a nonzero solution for $\widetilde{I}_{\text {int }, 1}(\widetilde{\rho})$.

\section{Numerical results}

We investigated Eq. (19) numerically for $\tau=1$ and with the density function $g$ defined as

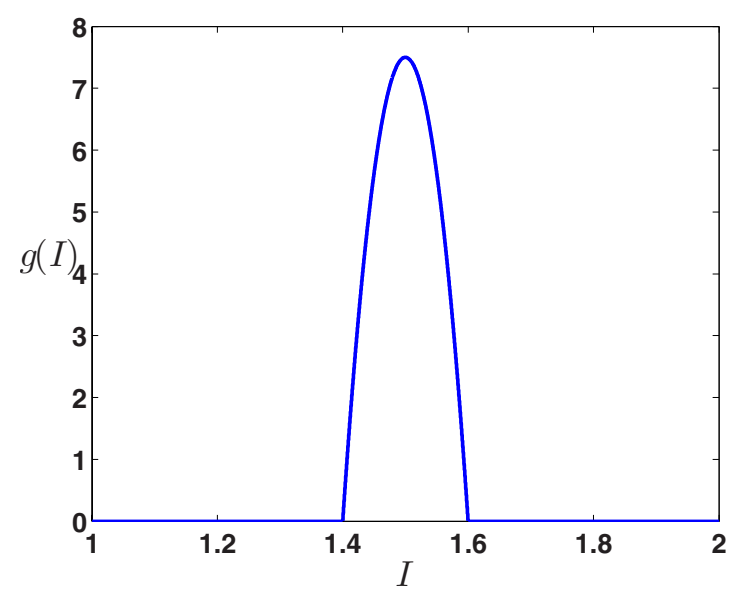

FIG. 2. (Color online) The density function $g$ used in the simulations.

$$
g(I) \triangleq \begin{cases}\frac{15}{2}\left[1-100\left(I-\frac{3}{2}\right)^{2}\right], & I \in\left[\frac{7}{5}, \frac{8}{5}\right] \\ 0, & I \notin\left[\frac{7}{5}, \frac{8}{5}\right] .\end{cases}
$$

The density function $g$ corresponds to a narrow unimodal distribution, centered at $\frac{3}{2}$; it is depicted in Fig. 2. It has a finite support, mainly because of the assumptions we imposed on $g$. [Recall that we have imposed $g(I)=0$ for $I$ $\leq 1 / \tau$, and that the average current $\int I g(I) d I$ is finite, implying that $g(I)$ should be decreasing sufficiently fast with $I$ for large $I$. This excludes, e.g., a Lorentz distribution.] A second motivation for considering a density function with finite support concerns the fact that the simulations (later on, in Sec. IV) rely on the finite system (1), which seems less appropriate as an approximation of the infinite system when $g$ has infinite support. [On the other hand, for fast decreasing density functions there may not be much difference concerning the numerical simulations, since the discrete values for $I$ in an approximation by the finite system may still be confined to a small interval. For example, the following numerical results remained qualitatively unaltered when replacing $g$ with a Gaussian with the same mean $\frac{3}{2}$ and the same value for $g\left(\frac{3}{2}\right)$.]

The simulation results are presented in Fig. 3. Figure 3(a) shows the eigenvalues $\lambda$ in the complex plane for varying $\epsilon$. [Only the part for which $\operatorname{Im}(\lambda) \geq 0$ is shown; since the complex conjugate values are also eigenvalues, there is symmetry about the real axis.] Figure 3(b) shows the real part of the eigenvalues as a function of $\epsilon$. It reveals that the real part may become nonzero above a threshold value $\epsilon_{\mathrm{A}}$ for $\epsilon$ about 0.208 .

Since the neutral stability of the linearized equations for $\epsilon<\epsilon_{\mathrm{A}}$ does not allow us to draw conclusions about the stability of the stationary solution in the original nonlinear system, we have carried out simulations using the finite system (1) for varying system sizes $N$. (See the Appendix for details.) The simulations show that, when the synchronous solution exists (see Sec. IV), the stationary solution is unstable, and synchronization emerges, also for $\epsilon<\epsilon_{\mathrm{A}}$. However, since 

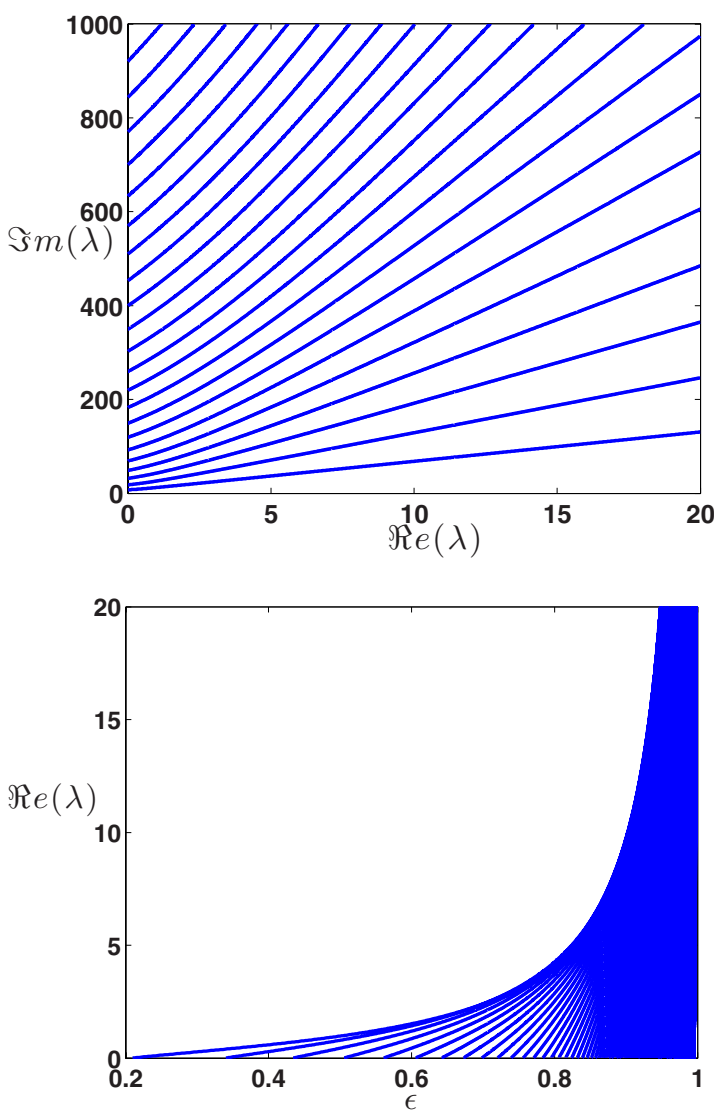

FIG. 3. (Color online) Eigenvalues of $\mathcal{L}$ for varying $\epsilon$; (a) projection onto the complex $(\operatorname{Re}(\lambda), \operatorname{Im}(\lambda))$ plane and (b) projection onto the $(\epsilon, \operatorname{Re}(\lambda))$ plane.

this may be a result of $N$ being finite, it may be that the time needed to reach synchronization becomes infinitely large for $N \rightarrow \infty$. We therefore investigated the evolution of the transient time with varying $N$, starting from a perturbed stationary solution.

We consider the times $T_{\text {half }}$ defined as the first time instance for which at least half of the population fired at a common time instance. (This is possible since the size of the synchronized group is more than half of the population in a broad range around $\epsilon \approx \epsilon_{\mathrm{A}}$ as is shown in Fig. 6 in Sec. IV.) It turns out that for a large range of $\epsilon$ values, the values $T_{\text {half }}$ depend on $N$ according to power laws. This is illustrated in Fig. 4, where the average $\left\langle\ln \left(T_{\text {half }}\right)\right\rangle$ of the values $\ln \left(T_{\text {half }}\right)$ over several simulation runs has been plotted in terms of $\ln (N)$. Since this behavior implies infinite $T_{\text {half }}$ values as $N$ goes to infinity, it is expected that this relationship can only be valid for $\epsilon<\epsilon_{\mathrm{A}}$. Indeed, for $\epsilon>\epsilon_{\mathrm{A}}$ the curves in Fig. 4 are no longer straight lines, but they become concave, in agreement with our analysis which predicts that the stationary solution is unstable for these values of $\epsilon$. In order to characterize the deviation from a power law, we consider the correlation coefficient $R$ of the values $\left\langle\ln \left(T_{\text {half }}\right)\right\rangle$ with respect to the values $\ln (N)$, as shown in Fig. 5. The values of $\epsilon$ for which $R$ is close to 1 correspond to a good agreement with a power law; a deviation from 1 indicates that the power law is not valid and (taking into account Fig. 4) that for $N \rightarrow \infty, T_{\text {half }}$ may remain finite. We find that the power law is valid for

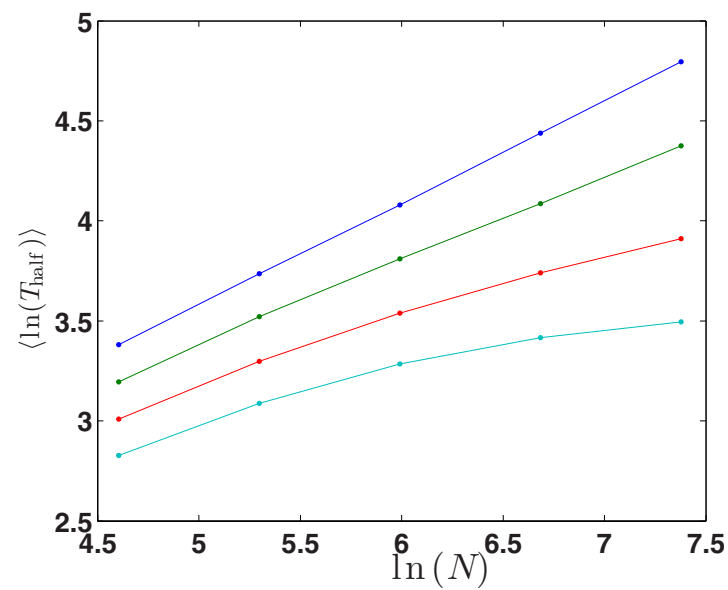

FIG. 4. (Color online) Graphs of $\left\langle\ln \left(T_{\text {half }}\right)\right\rangle$ in terms of $\ln (N)$ for different values of $\epsilon[\epsilon \in\{0.1813,0.1906,0.2008,0.2120\}$, with smaller $\left\langle\ln \left(T_{\text {half }}\right)\right\rangle$ values for larger values of $\left.\epsilon\right]$.

$\epsilon \lesssim \epsilon_{\mathrm{A}}$ and breaks down for $\epsilon \gtrsim \epsilon_{\mathrm{A}}$ (although the transition is not sharp enough to be able to estimate the value of $\epsilon_{\mathrm{A}}$ ). We conclude that the stationary state for the infinite model is stable-possibly only neutrally stable-for $\epsilon<\epsilon_{\mathrm{A}}$ and unstable for $\epsilon>\epsilon_{\mathrm{A}}$.

\section{Detailed transient behavior}

For $\epsilon<\epsilon_{\mathrm{A}}$ the instability of the stationary solution for finite $N$ may be explained as follows. In a rough approximation the membrane potential $U$ of a neuron with external input current $I$ may be considered as a random variable, distributed according to the stationary density function $\rho_{0}(U, I)$. When two neurons are not synchronized (i.e., firing simultaneously) we may consider their membrane potentials more or less as independently distributed random variables. Because of this, there is a nonzero probability that one of the two neurons has a membrane potential in the interval $\left[1-\frac{\epsilon}{N}, 1\right]$ when the other neuron fires. As a result they will fire simultaneously, and if their difference in external input current is sufficiently small with respect to $\epsilon / N$, they may remain synchronized during subsequent firings. (See also the condition

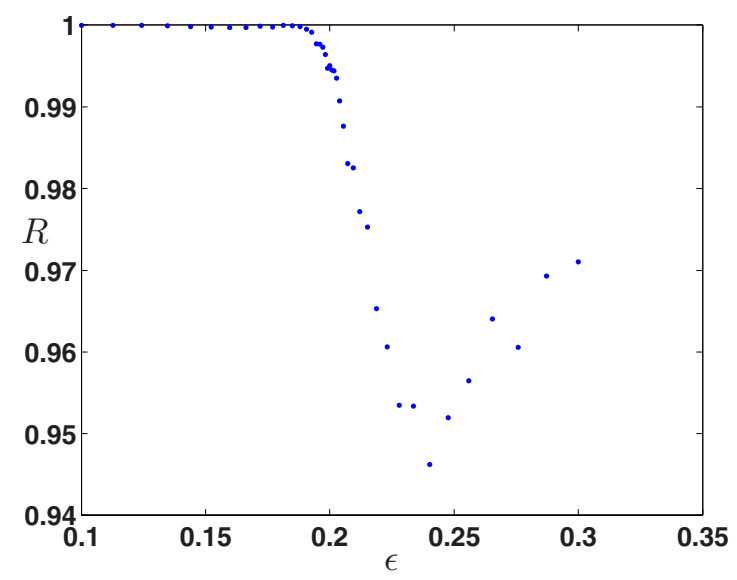

FIG. 5. (Color online) Correlation coefficient of $\left\langle\ln \left(T_{\text {half }}\right)\right\rangle$ with respect to $\ln (N)$ in terms of $\epsilon$. 
on $\epsilon$ for the existence of the synchronized solution in the next section.)

Consequently, for finite $N$ small synchronized groups of neurons may arise. A synchronized group of size $S$ may absorb a neuron with an external input current sufficiently close to the input currents of the neurons in the group if the membrane potential of this neuron at the given time instance belongs to the interval $\left[1-\frac{S}{N} \epsilon, 1\right]$, resulting in an absorption rate proportional to $S / N$. Since for a given size $S$ the number of neurons that may be considered for absorption is on the order of magnitude of $S$, the growth-regarded as a fraction of the population-is also inversely proportional to $N$.

Because of this, the growth of a synchronized group (starting from $S=2$ ) to a size that represents a significant fraction of $N$ decreases with increasing $N$ and may become infinitely slow for $N=\infty$. (We did not succeed in estimating the growth rate analytically by quantifying these observations due to the fact that the origination of several synchronized groups has to be considered, and the interaction between these groups may no longer be neglected.) Notice that one of the aspects governing the increase in the transient time with system size relates to the fact that the neurons have nonidentical input currents, in contrast to studies where the input currents are identical and large transient times arise as a result of a sparse network structure [29-31].

\section{PARTIALLY SYNCHRONOUS SOLUTION}

\section{A. Analytical results}

We call a solution (partially) synchronous if there is a group of neurons, constituting a nonzero fraction of the population (and therefore containing neurons with different external input currents) and firing at the same time instances. We will consider an analytical approximation by assuming that the neurons that do not belong to the synchronized group may be described by the stationary solution discussed in the previous section, even though one can easily see that the actual distribution will contain Dirac pulses as a consequence of the fact that when the synchronized group fires, all neurons with a value for $U$ in an interval below 1 will also fire, resulting in a Dirac pulse for $U=0$ that will propagate through the interval $[0,1]$ by dynamics (1). Taking these Dirac pulses into account results in quite complex behavior, and for convenience we will assume that the actual (timevarying) distribution is well approximated by $\rho_{0}$, introduced in the previous section.

We let $I_{\mathrm{u}}$ (respectively, $I_{1}$ ) be the maximal (respectively, minimal) external input current of the neurons in the synchronized group. We approximate the interaction current $I_{\text {int }}(t)$ by the constant value $I_{\text {int }, 0}$, implicitly defined by the following modification of Eq. (7):

$$
I_{\mathrm{int}, 0}=\epsilon \int_{\operatorname{supp}(g) \backslash\left[I_{1}, I_{\mathrm{u}}\right]} \frac{-g(I) d I}{\tau \ln \left(1-\frac{1}{\tau\left(I+I_{\mathrm{int}, 0}\right)}\right)} .
$$

Then we can easily calculate the time $T_{\mathrm{f}}$ a neuron with external input current $I_{\mathrm{u}}$ needs to reach the threshold $(U=1)$ by the equation

$$
\tau\left(I_{\mathrm{u}}+I_{\text {int }, 0}\right)\left(1-e^{T_{\mathrm{f}} / \tau}\right)=1 .
$$

On the other hand, a neuron with external input current $I$ $\in\left[I_{1}, I_{\mathrm{u}}\right)$, starting at rest (i.e., with $U=0$ ) at the same time instance as the neuron with external current $I_{\mathrm{u}}$, will not have fired yet, but it will have a value for $U$ equal to

$$
U=\tau\left(I+I_{\mathrm{int}, 0}\right)\left(1-e^{T_{\mathrm{f}} / \tau}\right) .
$$

If all neurons with external input in the interval $\left(I, I_{\mathrm{u}}\right]$ fire at this time instance, then the neuron with external input $I$ will also fire if

$$
\tau\left(I+I_{\text {int }, 0}\right)\left(1-e^{T_{\mathrm{f}} / \tau}\right)+\epsilon \int_{I}^{I_{\mathrm{u}}} d I^{\prime} g\left(I^{\prime}\right) \geq 1,
$$

or, using Eq. (23),

$$
\epsilon \int_{I}^{I_{\mathrm{u}}} d I^{\prime} g\left(I^{\prime}\right) \geq \frac{I_{\mathrm{u}}-I}{I_{\mathrm{u}}+I_{\mathrm{int}, 0}} .
$$

Considering the limit $I \rightarrow I_{\mathrm{u}}$ it follows that $\epsilon g\left(I_{\mathrm{u}}\right)\left(I_{\mathrm{u}}\right.$ $\left.+I_{\text {int }, 0}\right) \geq 1$. Since $I_{\mathrm{u}}$ corresponds to the maximal value of $I$ in the synchronized group, equality will hold (otherwise, the group may still grow), and $I_{\mathrm{u}}$ can be approximated as the largest solution of

$$
\epsilon g\left(I_{\mathrm{u}}\right)\left(I_{\mathrm{u}}+I_{\mathrm{int}, 0}\right)=1,
$$

The value of $I_{1}$ corresponds to the smallest value of $I$ such that Eq. (24) is satisfied for all $I$ in $\left[I_{1}, I_{\mathrm{u}}\right]$. As a result Eq. (24) will hold with equality for $I=I_{1}$ :

$$
\epsilon \int_{I_{1}}^{I_{\mathrm{u}}} d I^{\prime} g\left(I^{\prime}\right)=\frac{I_{\mathrm{u}}-I}{I_{\mathrm{u}}+I_{\text {int }, 0}} .
$$

This equation has to be considered simultaneously with Eqs. (25) and (22) because of the dependence of $I_{\text {int, }, 0}$ on $I_{1}$ and $I_{\mathrm{u}}$.

The size of the interval $\left[I_{1}, I_{\mathrm{u}}\right]$ will decrease with decreasing $\epsilon$ [simulations indicate that $I_{\mathrm{u}}$ will decrease and $I_{1}$ will increase, although this may not be immediately obvious from Eqs. (25) and (26) since $I_{\text {int, } 0}$ also depends on $\epsilon$ as well as on the values of $I_{1}$ and $I_{\mathrm{u}}$. The synchronized group has a (nonzero) minimal size; the corresponding value $\epsilon_{\mathrm{S}}$ for $\epsilon$ may be estimated as the smallest value of $\epsilon$ for which Eqs. (22), (25), and (26) have a solution for $I_{\mathrm{int}, 0}, I_{1}$, and $I_{\mathrm{u}}$.

\section{B. Numerical results}

We consider again $\tau=1$ and the density function $g$ defined by Eq. (21). As mentioned in Sec. III, most solutions of the finite system (1) converge to the synchronous solution when it exists. In Fig. 6 we plot the sizes $\alpha_{\mathrm{S}}$ of the synchronized group (as a fraction of the population) for different values of the coupling strength $\epsilon$, obtained by simulations with a network of 2000 neurons, as well as analytical estimations based on Eqs. (22), (25), and (26). Figure 6 only shows simulations corresponding to values of $\epsilon$ for which the synchronization appeared to be stable. For values of $\epsilon$ near $\epsilon_{\mathrm{S}}$ synchronization seemed to arise and to break down repeatedly, and those values have not been taken into account, although-as we will show in the next section-some of 


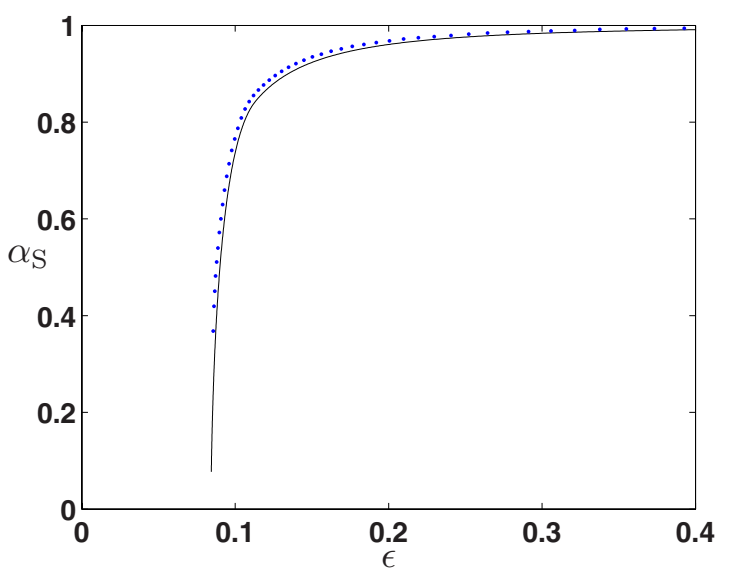

FIG. 6. (Color online) The fraction $\alpha_{\mathrm{S}}$ of neurons in the synchronized group in terms of $\epsilon$, obtained by simulations with $N$ $=2000$ (dots) and by the analytical estimation using Eqs. (22), (25), and (26) (solid line).

them may also correspond to stable synchronization when considering the limit $N \rightarrow \infty$.

There is a considerable gap between analysis and simulations regarding the minimal value of $\alpha_{\mathrm{S}}$, which may be attributed, on one hand, to these missing data points and, on the other hand, to the approximate character of the analysis. For the estimation of the threshold value $\epsilon_{\mathrm{S}} \approx 0.086$ there is a good agreement between simulations and analysis.

\section{SELF-REPEATING BEHAVIOR FOR FINITE $N$}

\section{A. Results}

Simulations (of the finite model) show that there is a small region for $\epsilon$ around $\epsilon_{\mathrm{S}}$ (corresponding approximately to the interval $[0.0841,0.0856])$, where the solution is neither stationary nor synchronous. The solution seems to build up toward synchronization, and when a particular level of synchrony is attained, the synchrony suddenly breaks down, after which the process is repeated. A typical solution in this range for $\epsilon$ is shown in Fig. 7.

When $N$ is increased, the time needed to build up the synchronization again increases according to a power law with $N$, and similarly as in Sec. III we conclude that the stationary solution is stable for $N \rightarrow \infty$. To some extent, a similar reasoning holds for the synchronous solution.

Even though the breakdown time seems to be relatively small in Fig. 7, simulations show that it also increases with $N$ for some (sufficiently large) values of $\epsilon$, indicating the existence of a stable synchronous solution. For other values of $\epsilon$ we were unable to draw firm conclusions; with increasing $N$ the breaking down of the synchronization seems to become more gradual, and the corresponding time interval is not well defined, while the maximal value of $\alpha_{\mathrm{S}}$ still seems to increase with $N$ for the values of $N$ we used in the simulations (in the range $10^{4}-10^{5}$ ). Since we were not able to consider larger values of $N$ (the corresponding simulation times became too large), it remains unclear whether the synchronous solution exists for these values of $\epsilon$ and for infinite $N$. We conclude that the actual value of $\epsilon_{\mathrm{S}}$ most likely belongs to

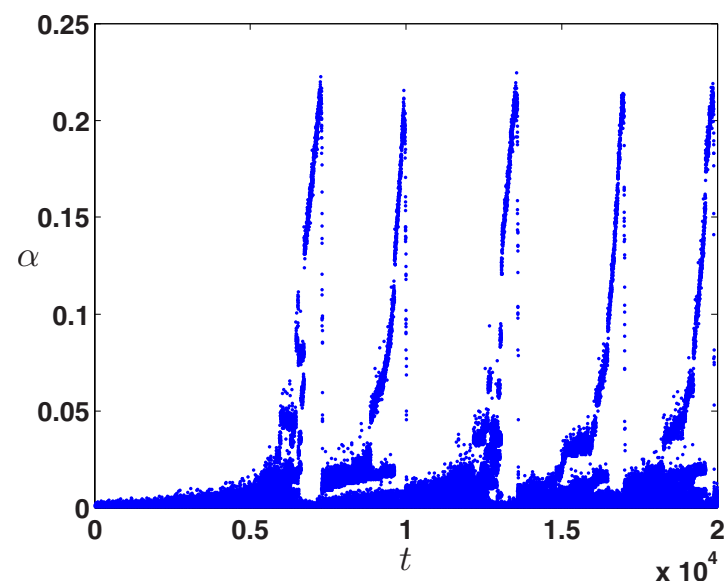

FIG. 7. (Color online) Simulation of a solution exhibiting neither stationary nor synchronous behavior, corresponding to $\epsilon$ $=0.085$. The fraction $\alpha$ of simultaneously spiking neurons is plotted as a function of time. (Notice that this also includes the spikes of the neurons that are not in the synchronized group, which account for the largest part of the data and correspond to small values for $\alpha$.)

the interval [0.0841, 0.0856], but simulations with larger $N$ would be needed to provide a more accurate estimation.

\section{B. Detailed behavior}

The self-repeating behavior may be explained as follows by considering Eqs. (22), (25), and (26) for $\epsilon \lesssim \epsilon_{\mathrm{S}}$, and assuming that $I_{\text {int, } 0}, I_{\mathrm{u}}$, and $I_{1}$ vary accordingly. Starting from asynchronous behavior, the solution $I_{\text {int, } 0}$ to Eq. (22) will be maximal [and corresponding to the solution of Eq. (7)], and such that Eq. (25) has two solutions for $I_{\mathrm{u}}$ (there may be more solutions for other distributions $g$, but for a typical unimodal $g$ there will only be two solutions). Neurons with $I$ values in between these two solutions may synchronize, and from then on the synchronized group may grow (mainly toward smaller $I$ values) by adding neurons with $I$ values in the interval $\left[I_{1}, I_{\mathrm{u}}\right]$ [with $I_{1}$ defined by Eq. (26), and $I_{\mathrm{u}}$ being the largest solution of Eq. (25)]. As the synchronized group grows, the group of neurons contributing to the interaction current decreases, and $I_{\text {int, } 0}$ will decrease according to Eq. (22) (with $\left[I_{1}, I_{\mathrm{u}}\right]$ replaced with the set of $I$ values of the neurons that actually belong to the synchronized group). As $I_{\text {int, } 0}$ decreases, the solution $I_{\mathrm{u}}$ of Eq. (25) will decrease, and therefore some neurons may leave the synchronized group as their $I$ value becomes too large. As long as the synchronized group does not contain all neurons with $I$ values in the interval $\left[I_{1}, I_{\mathrm{u}}\right]$, it will continue to grow and $I_{\text {int }, 0}$ will continue to decrease. Since we assume $\epsilon<\epsilon_{\mathrm{S}}$, there will be a group size (smaller than the size corresponding to the entire interval $\left[I_{1}, I_{\mathrm{u}}\right]$ ) for which the corresponding $I_{\mathrm{int}, 0}$ is such that Eq. (25) no longer has a solution for $I_{\mathrm{u}}$. At that point, the synchronized group will break down entirely, leading to a sudden increase in $I_{\text {int }, 0}$ to its maximal value, after which the process is repeated.

When $\epsilon$ decreases, the maximal size for the synchronized group will decrease, until the initial value of $I_{\text {int, } 0}$ [i.e., the solution of Eq. (7)] is too small and Eq. (25) has no solutions 
for $I_{\mathrm{u}}$. In this case the solutions of the finite model (with $N$ sufficiently large) are well described by the stationary density function $\rho_{0}$ (also for large $t$ ).

For $\epsilon \gtrsim \epsilon_{\mathrm{S}}$ a similar reasoning holds, but the breakdown of the synchronized group may be attributed to temporal finitesize fluctuations in $I_{\text {int }, 0}$ as the size of the synchronized group approaches its maximal value. Consider, e.g., the scenario where, due to a small fluctuation in $I_{\text {int, } 0}$, the fastest neuron in the synchronized group fires ahead of the group. The difference in fire times between this neuron and the remainder of the group is related to the fluctuation size. However, at the next firing time this difference will have grown due to the interaction exerted by the remainder of the synchronized group on this neuron. (This interaction did not occur when the neuron belonged to the synchronized group because of the refractoriness.) As a result the separation between the neuron and the synchronized group is no longer related to the fluctuation size and cannot be compensated by an opposite fluctuation. Repetition of this scenario leads to a breakdown of the synchronized group, with a breakdown time that grows with $N$ (since with increasing $N$ larger fluctuations occur less frequently, and the number of synchronized neurons increases).

\section{CONCLUSION}

We have considered an all-to-all excitatorily coupled neural network consisting of an infinite number of leaky integrate-and-fire neurons. In contrast with most systems considered in the literature, the neurons have different external input currents, assigned according to a unimodal distribution. The analytical results show that stationary behavior becomes unstable for values of the coupling strength above a threshold value. Below this value, simulations indicate that the stationary solution may be stable, since the time span during which the solution retains its stationary character increases with the (finite) system size according to a power law.

There is also a lower threshold below which no synchronization is possible. Its value can be estimated analytically. Above this threshold (except for a small neighborhood) simulations with a finite number of neurons indicate that the (partially) synchronized solution is stable. In between the two threshold values, both the stationary and the synchronized solutions exist and are stable for the infinite model. The existence of a parameter region where the system exhibits bistability is similar to the results of [28], where an effec- tive inhibitory interaction is considered in this regard.

For the finite model, a solution starting from a perturbation of a stationary solution will eventually (partially) synchronize (for values of the coupling strength above the lower threshold), but the transient time grows unbounded with increasing system size as a result of the dispersity in external input currents (as opposed to, e.g., the long transients studied in [29-31], which result from the sparsity of the network).

In a small range for the coupling strength in a neighborhood of the lower threshold, the solutions of the finite model are neither stationary nor synchronous, but synchronization is built up and breaks down again repeatedly. In the limit of an infinite number of neurons, these solutions may also be classified as either stationary or synchronous.

\section{ACKNOWLEDGMENTS}

This paper presents research results of the Belgian Network Dynamical Systems, Control, and Optimization (DYSCO), funded by the Interuniversity Attraction Poles Programme, initiated by the Belgian State, Science Policy Office. This research was supported by the Research Foundation-Flanders (FWO) through Project No. G.0026.06N.

\section{APPENDIX: SIMULATION DETAILS}

For the simulation of a finite number of neurons governed by Eq. (1), we chose to define the external input currents $I_{i}$ $(i \in\{1, \ldots, N\})$ by the density function $g$ according to the following equation:

$$
\int_{-\infty}^{I_{i}} g(I) d I=\frac{i-\frac{1}{2}}{N},
$$

resulting in a better correspondence between the values $I_{i}$ and the distribution $g$ than when the currents $I_{i}$ would have been drawn randomly from $g$. The initial membrane potential values $U_{i}(0)$ were drawn randomly from distribution (5), with $I_{\text {int, } 0}$ approximated by Eq. (9). For the simulations carried out to calculate $T_{\text {half }}$, the initial membrane potential for each tenth neuron (when ordered according to increasing external input current) was put equal to zero to provide a similar perturbation for the different simulation runs. The parameter $\tau$ was set equal to 1 . The simulation of the dynamics was done exactly, using the analytical solution of Eq. (1) instead of a numerical integration algorithm.
[1] J. J. Hopfield, Proc. Natl. Acad. Sci. U.S.A. 79, 2554 (1982).

[2] R. P. N. Rao and T. J. Sejnowski, Philos. Trans. R. Soc. London, Ser. A 361, 1149 (2003).

[3] B. L. McNaughton, F. P. Battaglia, O. Jensen, E. I. Moser, and M.-B. Moser, Nat. Rev. Neurosci. 7, 663 (2006).

[4] Y. Choe and R. Miikkulainen, Neurocomputing 21, 139 (1998).
[5] S. Tanaka, M. Miyashita, and J. Ribot, Neural Networks 17, 1363 (2004).

[6] M. Lengyel, Z. Huhn, and P. Érdi, Biol. Cybern. 92, 393 (2005).

[7] S. M. Bohte, H. L. Poutré, and J. N. Kok, IEEE Trans. Neural Netw. 13, 426 (2002).

[8] A. Delorme and S. J. Thorpe, Neural Networks 14, 795 
(2001).

[9] A. Plebe and R. G. Domenella, Neural Networks 20, 763 (2007).

[10] F. Wörgötter and B. Porr, Neural Comput. 17, 245 (2005).

[11] S. J. Thorpe and M. Imbert, Connectionism in Perspective (Elsevier, New York, 1989), pp. 63-92.

[12] S. Thorpe, D. Fize, and C. Marlot, Nature (London) 381, 520 (1996).

[13] J. O'Keefe and M. L. Recce, Hippocampus 3, 317 (1993).

[14] Ch. von der Malsburg, Max-Planck-Institut für Biophysikalische Chemie Internal Report No. 81-2, 1981 (unpublished); reprinted in Models of Neural Networks II, edited by E. Domany, J. L. van Hemmen, and K. Schulten (SpringerVerlag, Berlin, 1994), Chap. 2, pp. 95-119.

[15] Ch. von der Malsburg and W. Schneider, Biol. Cybern. 54, 29 (1986).

[16] R. Eckhorn, R. Bauer, W. Jordan, M. Brosch, W. Kruse, M. Munk, and H. J. Reitboeck, Biol. Cybern. 60, 121 (1988).

[17] R. Eckhorn, A. M. Gail, A. Bruns, A. Gabriel, B. Al-Shaikhli, and M. Saam, IEEE Trans. Neural Netw. 15, 1039 (2004).

[18] P. J. Uhlhaas and W. Singer, Neuron 52, 155 (2006).

[19] R. E. Mirollo and S. H. Strogatz, SIAM J. Appl. Math. 50, 1645 (1990).

[20] M. Timme and F. Wolf, Nonlinearity 21, 1579 (2008).

[21] W. Gerstner, Phys. Rev. E 51, 738 (1995).

[22] C. van Vreeswijk, Phys. Rev. E 54, 5522 (1996).
[23] K. A. Newhall, G. Kovačič, P. R. Kramer, D. Zhou, A. V. Rangan, and D. Cai, Commun. Math. Sci. 8, 541 (2010).

[24] C. S. Peskin, Mathematical Aspects of Heart Physiology (Courant Institute of Mathematical Sciences, New York University, New York, 1975).

[25] B. W. Knight, J. Gen. Physiol. 59, 734 (1972).

[26] M. Tsodyks, I. Mitkov, and H. Sompolinsky, Phys. Rev. Lett. 71, 1280 (1993).

[27] W. Senn and R. Urbanczik, SIAM J. Appl. Math. 61, 1143 (2001).

[28] S. Ostojic, N. Brunel, and V. Hakim, J. Comput. Neurosci. 26, 369 (2009).

[29] A. Zumdieck, M. Timme, T. Geisel, and F. Wolf, Phys. Rev. Lett. 93, 244103 (2004).

[30] R. Zillmer, R. Livi, A. Politi, and A. Torcini, Phys. Rev. E 74, 036203 (2006).

[31] R. Zillmer, N. Brunel, and D. Hansel, Phys. Rev. E 79, 031909 (2009).

[32] C. van Vreeswijk and H. Sompolinsky, Science 274, 1724 (1996).

[33] C. van Vreeswijk and H. Sompolinsky, Neural Comput. 10, 1321 (1998).

[34] A. W. Naylor and G. R. Sell, Linear Operator Theory in Engineering and Science, Applied Mathematical Sciences Vol. 40 (Springer-Verlag, New York, 1982). 